\title{
Ectatic Coronary Vasculopathy in Homocygous Alpha-1 Antitrypsin Deficiency
}

\author{
Drina Petener ${ }^{\mathrm{a}, \mathrm{c}}$, Jutta Bergler-Klein ${ }^{\mathrm{b}}$, Franz Weidinger ${ }^{\mathrm{a}}$, Paul Wexberg ${ }^{\mathrm{a}}$
}

\begin{abstract}
We report on a 45-year old male who underwent evaluation for lung-transplantation because of end-stage chronic obstructive pulmonary disease due to homozygous alpha-1-antitrypsin-deficiency. Routine coronary angiography at that time was normal, however, the patient did not consent to the operation then and two years later all examinations were repeated, when the clinical condition had worsened. Repeated angiography now revealed a severe ectatic coronary vasculopathy. We hypothesize that the simultaneous effect of elastine-degraduation in arterial walls by uninhibited activity of proteolytic elastase and constant hypoxemia due to the patient's progressive lung disease caused the rapid development of coronary aneurysms.
\end{abstract}

Keywords: Ectatic; Coronary; Vasculopathy; Alpha-1-Antitrypsin; Deficiency

\section{Introduction}

Alpha1-Antitrypsin (AAT) deficiency causes overreaching activity of neutrophil elastase in blood and lungs and thus an increased risk of panacinar emphysema and COPD (chronic obstructiv lung disease) especially among smokers, in addition to hepatic cirrhosis and vascular aneurysms. The deficiency is treated by avoidance of damaging inhalants, by intravenous infusions of the AAT protein and by transplantation of the lungs or liver [1]. AAT deficiency causes dis-

Manuscript accepted for publication January 2, 2013

\footnotetext{
and Department of Medicine, KA Rudolfstiftung, Vienna, Austria

${ }^{\mathrm{b}}$ Department of Internal Medicine II, Division of Cardiology,

Medical University of Vienna, Vienna, Austria

${ }^{\mathrm{c} C}$ Corresponding author: Drina Petener, 2nd Department of Medicine,

Rudolfstiftung, Juchgasse 25, A-1030 Vienna, Austria.

Email: drina.petener@wienkav.at
}

doi: http://dx.doi.org/10.4021/jmc1031w ability and considerably reduces life expectancy. We believe that there is a correlation between AAT deficiency and the development of ectatic coronary vasculopathy, which has not been described before. Equally coronary ectasia might give a hint to underlying connective tissue disease.

\section{Case Report}

We report a 45 year old male who was evaluated for lung transplantation because of end-stage COPD with long term oxygen therapy as a result of homocygous AAT-deficiency. Dyspnea on exertion had started 2000 at an age of 35. Massive emphysema had been diagnosed 2004, when the patient was in hospital because of severe pulmonal infection with Moraxella catarrhalis. His maximal walking distance without pausing then was $500 \mathrm{~m}$. Spiroergometry showed severe obstruction. Blood oxygen saturation was $90 \%$ at rest and $84 \%$ on exertion $\left(\mathrm{pO}_{2}\right.$ in capillary blood $7.45 \mathrm{kPa}$ [56 Torr] at rest, $6.40 \mathrm{kPa}$ [48 Torr] on exertion). The patient then quit smoking after 25 pack-years. Homocygous AAT-deficiency (Pi-ZZ allels) was detected and intravenous AAT-augmentation therapy ( $4 \mathrm{~g}$ Prolastin ${ }^{\circledR}$ per week) started after confirmation of AAT $<11 \mu \mathrm{mol} / \mathrm{l}$. The first evaluation for lung transplantation took place in 2008 , when the initial coronary angiography was performed as a matter of routine before transplantation, which showed normal findings (Fig. 1). The result of spirometry again was severe obstruction without salbutamol-effect (RV 6.00 1, TLC 9.62 1, ITGV 6.44 1, FEV1 1.261 , FEV1 \% FVC 36.33). Nevertheless the patient did not consent to the operation at that time.

From that time on he had been receiving long-term oxygen therapy for all day. The patient's condition remained stable until spring 2010, when he experienced dyspnoea on minimal exertion like walking 20 meters, and all essential examinations for lung transplantation were repeated. Capillary partial pressure of oxygen was $8.00 \mathrm{kPa}$ [60 Torr] while breathing ambient air at rest. The spirometry had worsened again (RV 6.83 1, TLC 9.36 1, ITGV 7.96 1, FEV1 0.81 1, FEV1 \% FVC 32.01). Computed tomography of the thorax had not changed within the last 2 years. Electrocardiogram and echocardiogram were normal except for signs of right 

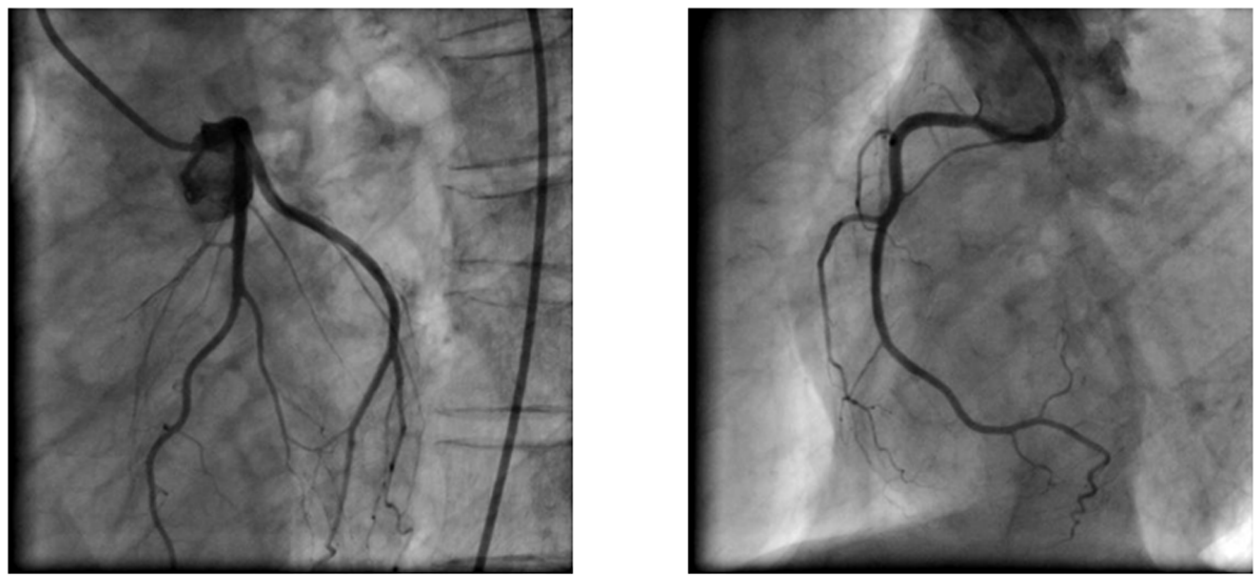

Figure 1. Baseline angiogram at the time of first transplant evaluation. Normal left and right coronary artery.

heart hypertrophy with still good right ventricular function. Surprisingly the repeated coronary angiography revealed that within only two years an ectatic coronary vasculopathy had developed, predominantly in the proximal and mid left anterior discending artery. Mild ectasia was also found in the distal right coronary artery (Fig. 2). No ectasia or aneurysm was detected in any other investigation such as contrastagent CT of the aorta or duplex sonography of the carotides. After dismission from our hospital the patient was further treated at the University Hospital in preparation for the lungs transplantation.

\section{Discussion}

AAT is the most important inhibitor of serine proteases (for example elastase, chymotrypsin, and collagenase) in the blood (90\%). AAT is an acute-phase reactant. Its deficiency causes overreaching activity of neutrophil elastase in blood and lungs and thus an increased risk of panacinar emphysema and COPD (chronic obstructive pulmonary disease) especially among smokers. In liver cells, where the defective synthesis of AAT takes place, the increased deposition of polymerized AAT molecules in cytoplasmatic globules causes liver disease.

The AAT-gene is localized on chromosome 14q 31 - 33.2. Several degrees of deficiency are known: The homocygous type (phenotype PIZZ or PISS) has an incidence of 1 case per 10000 persons, which is similar to that of cystic fibrosis. The AAT-concentration is below $50 \mathrm{mg} / \mathrm{dl}$ (usually $15 \%$ of normal range). The main symptoms of the severe, homocygous type are prolonged neonatal obstructive jaundice, earlyonset panacinar emphysema, sometimes bronchiectasis and eventually hepatocirrhosis and hepatocellular carcinoma. Some infants undergo liver-transplantation because of organ failure. COPD is rarely seen below the age of 30 years.
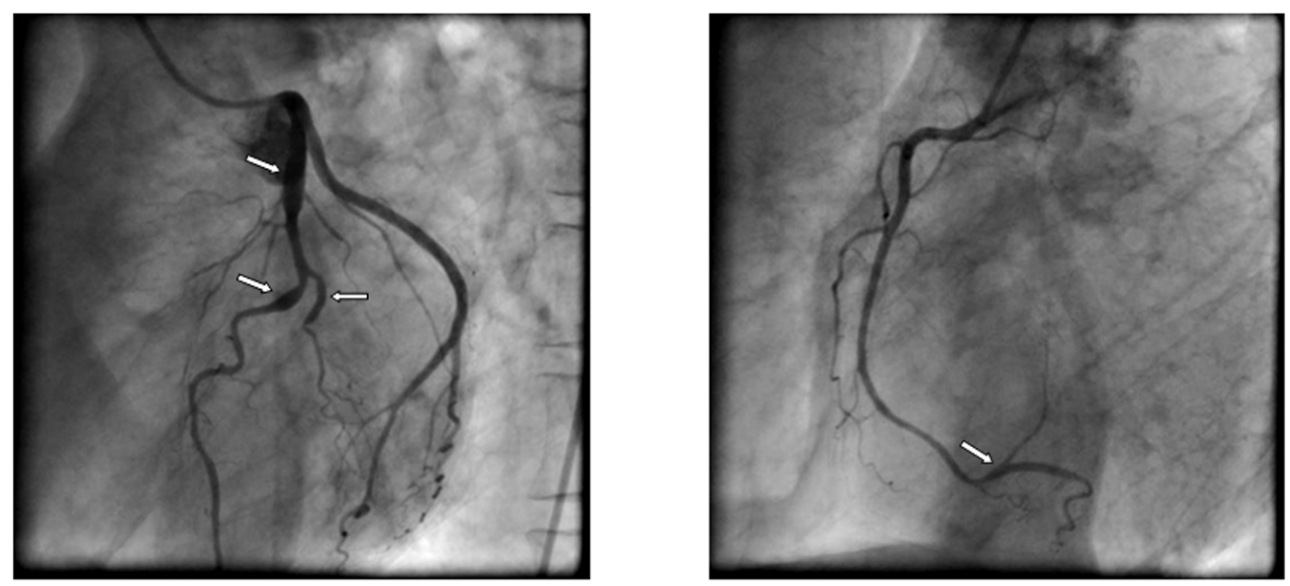

Figure 2. Follow-up angiogram two years later. Note the ectatic segments (arrows) in the proximal and mid left-anterior descending artery, the 2 nd diagonal branch and the distal right coronary artery. 
In the heterocygous type (phenotype PIMZ or PIMS) the AAT-concentration is $50-250 \mathrm{mg} / \mathrm{dl}$. The risk for COPD and liver disease is increased in the PIMZ-genotype group, especially in smokers. About 1\% of all COPD patients have AAT deficiency. The diagnosis is usually made up after that of COPD or liver disease or by screening of the family after the deficiency has been diagnosed in a relative. A hint to the diagnosis is the decreased fraction of alpha 1 globulins in protein electrophoresis. It is affirmed by decreased AAT serum level, AAT-genotyping and liver biopsy (AAT deposits in hepatocytes).

The deficiency is treated by avoidance of damaging inhalants. Medicinal treatment of AAT deficiency is equal to therapy of COPD, guided by severity of the lung-disease. Furthermore vaccination against pneumococcal infection and influenza is recommended. For end stage patients surgical lung volume reduction and uni- or bilateral lung transplantation are possible therapeutic options. Early antibiotic treatment in suspected bacterial respiratory infection to minimize the neutrophil burden of the lung is recommended. Intravenous AAT-augmentation therapy for COPD patients with serum levels of AAT $<11 \mu \mathrm{mol} / 1$ is indicated as lifelong treatment. For liver disease vaccination against hepatitis A and $\mathrm{B}$ are advisable. The therapy of portal hypertension and liver failure are the same as for liver diseases (low-protein diet, alcohol cessation, medical and endoscopic treatment of esophageal varices, portosystemic shunts, liver-transplantation).

We have described the development of coronary aneurysms within two years in a patient with homocygous AAT deficiency. Arterial aneurysms and spontaneous dissections have been found in various vascular districts $[2,3]$, but to the best of our knowledge coronary ectatic vasculopathy has not been described in AAT deficiency previously. The affection of vessels might be explained by the degradation of elastic vascular fibres by enhanced activity of neutrophil elastase which can disrupt the integrity of the vessel wall and cause the formation of ectasia and/or aneurysm. Alternatively, constant hypoxemia due to the patient's lung disease might have caused aneurysm formation: Firstly, coronary dilation is regulated independent from the endothelium by arterial pressure, myocardial metabolism and the autonomic nervous system as well as by arterial oxygen-saturation [4]. Secondly, low blood oxygen saturation stimulates coronary vasodilatation in angiographically normal coronary arterial segments, whereas it does not affect vascular diameters in atherosclerotic segments [5]. In our patient, chronic hypoxemia might have boosted the rapid development of ectatic coronary angiopathy in a setting of increased proteolytic elastase-activity due to AAT deficiency.

Our report emphasizes that asymptomatic development of coronary and other aneurysms should be considered during the management of AAT1 deficiency. The clinical management of these patients requires further investigation. On the other hand the detection of coronary aneurysms might point to connective tissue disease.

\section{References}

1. Silverman EK, Sandhaus RA. Clinical practice. Alpha1antitrypsin deficiency. NEngl J Med. 2009;360(26):27492757.

2. Schievink WI, Katzmann JA, Piepgras DG. Alpha-1-antitrypsin deficiency in spontaneous intracranial arterial dissections. Cerebrovasc Dis. 1998;8(1):42-44.

3. Gaglio PJ, Regenstein F, Slakey D, Cheng S, Takiff H, Rinker R, Dick D, et al. Alpha-1 antitrypsin deficiency and splenic artery aneurysm rupture: an association? Am J Gastroenterol. 2000;95(6):1531-1534.

4. Larsen BT, Gutterman DD. Hypoxia, coronary dilation, and the pentose phosphate pathway. Am J Physiol Heart Circ Physiol. 2006;290(6):H2169-2171.

5. Arbab-Zadeh A, Levine BD, Trost JC, Lange RA, Keeley EC, Hillis LD, Cigarroa JE. The effect of acute hypoxemia on coronary arterial dimensions in patients with coronary artery disease. Cardiology. 2009;113(2):149154. 